MATHEMATICS OF COMPUTATION

Volume 66, Number 217, January 1997, Pages 289-296

S 0025-5718(97)00794-1

\title{
SPHERICAL BESSEL FUNCTIONS AND EXPLICIT QUADRATURE FORMULA
}

\author{
RIADH BEN GHANEM AND CLÉMENT FRAPPIER
}

\begin{abstract}
An evaluation of the derivative of spherical Bessel functions of order $n+\frac{1}{2}$ at its zeros is obtained. Consequently, an explicit quadrature formula for entire functions of exponential type is given.
\end{abstract}

\section{Introduction And Statement of the RESUlts}

Given any complex number $\alpha$, the function

$$
\frac{J_{\alpha}(z)}{z^{\alpha}}=\sum_{k=0}^{\infty}(-1)^{k} \frac{z^{2 k}}{2^{\alpha+2 k} k ! \Gamma(k+\alpha+1)}
$$

is an even entire function of exponential type 1. Here $J_{\alpha}(z)$ is the Bessel function of the first kind of order $\alpha$ and is known as the spherical Bessel function when $\alpha=n+\frac{1}{2}, n \in \mathbb{Z}$. Let $j_{k}=j_{k}(\alpha), k= \pm 1, \pm 2, \ldots$, be the zeros of $\frac{J_{\alpha}(z)}{z^{\alpha}}$ ordered such that $j_{-k}=-j_{k}$ and $0<\left|j_{1}\right| \leq\left|j_{2}\right| \leq \ldots$.

An exact quadrature formula with zeros of Bessel functions as nodes has been recently given [1] as follows.

Theorem A. Let $\Re(\alpha)>-1$. For all functions $f$ of exponential type $2 \tau$ such that $f(x)=O\left(|x|^{-\delta}\right), x \rightarrow \pm \infty$, with $\delta>2 \Re(\alpha)+2$, we have

(1)

$$
\int_{0}^{\infty} x^{2 \alpha+1}(f(x)+f(-x)) d x=\frac{2}{\tau^{2 \alpha+2}} \sum_{k=1}^{\infty} \frac{j_{k}^{2 \alpha}}{\left(J_{\alpha}^{\prime}\left(j_{k}\right)\right)^{2}}\left(f\left(\frac{j_{k}}{\tau}\right)+f\left(-\frac{j_{k}}{\tau}\right)\right) .
$$

The growth condition imposed on the functions has been relaxed by Grozev and Rahman.

Theorem B ([2]). If $\alpha>-1$, then (1) holds for every entire function $f$ of exponential type $2 \tau$ such that $x^{2 \alpha+1}(f(x)+f(-x))$ belongs to $L^{1}[0, \infty)$.

Since, in formula $(1), J_{\alpha}^{\prime}\left(j_{k}\right)$ is not given explicitly, we find it interesting to evaluate it for the spherical Bessel functions. From now on, the notation $j_{k}$ is used exclusively to denote $j_{k}\left(n+\frac{1}{2}\right)$.

Received by the editor October 6, 1995 and, in revised form, January 26, 1996.

1991 Mathematics Subject Classification. Primary 33C10, 41A55; Secondary 65D32.

Key words and phrases. Bessel functions, quadrature formula, entire functions.

The research of the second author was supported by the Natural Sciences and Engineering Research Council of Canada Grant No. OGP 0009331. 
Theorem 1. Let $n$ be a nonnegative integer and

$$
\lambda\left(j_{k}\right):=\left(\frac{\pi}{2} \sum_{r=0}^{n} \frac{(2 n-r) !(2 n-2 r) !}{r !\left[2^{n-r}(n-r) !\right]^{2}} j_{k}^{2 r}\right)^{-\frac{1}{2}} .
$$

We have

$$
J_{n+\frac{1}{2}}^{\prime}\left(j_{k}\right)=(-1)^{k} j_{k}^{n-\frac{1}{2}} \lambda\left(j_{k}\right) \quad \text { for } \quad k= \pm 1, \pm 2, \ldots .
$$

Since (2) is not valid for negative integers, we give another result for these values.

We note that the zeros of $J_{\alpha}(z)$ are all real if $\alpha>-1$ and only a finite number of them are nonreal if $\alpha \leq-1$ [3, $\S 15.27]$. Let $\left\{l_{k}\right\}_{k=1}^{\infty}$ be the positive zeros of $\frac{J_{\alpha}(z)}{z^{\alpha}}, \alpha=n+\frac{1}{2}$, arranged in ascending order of magnitude and $l_{k}=-l_{-k}$ for $k=-1,-2, \ldots$.

Theorem 2. Let $n$ be a negative integer and

$$
\mu\left(l_{k}\right):=\left(\frac{\pi}{2} \sum_{r=0}^{-n-1} \frac{(-2 n-r-2) !(-2 n-2 r-2) !}{r !\left[2^{-n-r-1}(-n-r-1) !\right]^{2}} l_{k}^{2 r}\right)^{-\frac{1}{2}} .
$$

We have

$$
J_{n+\frac{1}{2}}^{\prime}\left(l_{k}\right)=\left\{\begin{array}{lll}
(-1)^{n+k+1} l_{k}^{-n-\frac{3}{2}} \mu\left(l_{k}\right) & \text { for } \quad k=1,2, \ldots, \\
(-1)^{n+k} l_{k}^{-n-\frac{3}{2}} \mu\left(l_{k}\right) & \text { for } \quad k=-1,-2, \ldots .
\end{array}\right.
$$

Remark 1. Using Theorems 1, 2 and the differential equation

$$
z^{2} y^{\prime \prime}+z y^{\prime}+\left(z^{2}-\alpha^{2}\right) y=0
$$

satisfied by $J_{\alpha}(z)$, we can evaluate $J_{n+\frac{1}{2}}^{\prime \prime}\left(j_{k}\right), J_{n+\frac{1}{2}}^{\prime \prime \prime}\left(j_{k}\right)$, etc.

\section{LEMMAS}

For the recurrence formulas satisfied by Bessel functions and used in this section we refer the reader to $[3, \S 3.2]$. We need the following property of spherical Bessel functions to prove formula (2).

Lemma 1. Let $n$ be an integer. For all nonnegative integers $p$, we have

$$
J_{n-p-\frac{1}{2}}\left(j_{k}\right)=\left\{\sum_{r=0}^{[p / 2]}(-1)^{r}\left(\begin{array}{c}
p-r \\
r
\end{array}\right) \frac{\Gamma\left(n-r+\frac{1}{2}\right) 2^{p-2 r}}{\Gamma\left(n-p+r+\frac{1}{2}\right) j_{k}^{p-2 r}}\right\} J_{n+\frac{1}{2}}^{\prime}\left(j_{k}\right) .
$$

Proof. We prove (4) by induction on $p$. For $p=0,(4)$ is equivalent to

$$
J_{n-\frac{1}{2}}\left(j_{k}\right)=J_{n+\frac{1}{2}}^{\prime}\left(j_{k}\right)
$$

which we obtain using the formula

$$
z J_{\alpha}{ }^{\prime}(z)+\alpha J_{\alpha}(z)=z J_{\alpha-1}(z)
$$

with $\alpha=n+\frac{1}{2}$ and $z=j_{k}$. For $p=1$, (4) gives $J_{n-\frac{3}{2}}\left(j_{k}\right)=\frac{2 n-1}{j_{k}} J_{n+\frac{1}{2}}^{\prime}\left(j_{k}\right)$, which is true by the formula

$$
J_{\alpha-1}(z)=\frac{2 \alpha}{z} J_{\alpha}(z)-J_{\alpha+1}(z),
$$

taking $\alpha=n-\frac{1}{2}$ and using (5). Suppose that (4) is true for $p$ and $p+1$, where $p$ is an even integer, and let us prove it for $p+2$ and $p+3$. 
When $\alpha=n-p-\frac{3}{2},(7)$ and the recurrence hypothesis give

$$
\begin{aligned}
J_{n-p-\frac{5}{2}}\left(j_{k}\right)= & \frac{2 n-(2 p+3)}{j_{k}} J_{n-p-\frac{3}{2}}\left(j_{k}\right)-J_{n-p-\frac{1}{2}}\left(j_{k}\right) \\
= & \left\{(2 n-2 p-3) \sum_{r=0}^{p / 2}(-1)^{r}\left(\begin{array}{c}
p+1-r \\
r
\end{array}\right) \frac{\Gamma\left(n-r+\frac{1}{2}\right) 2^{p+1-2 r}}{\Gamma\left(n-p+r-\frac{1}{2}\right) j_{k}^{p+2-2 r}}\right. \\
& \left.-\sum_{r=0}^{p / 2}(-1)^{r}\left(\begin{array}{c}
p-r \\
r
\end{array}\right) \frac{\Gamma\left(n-r+\frac{1}{2}\right) 2^{p-2 r}}{\Gamma\left(n-p+r+\frac{1}{2}\right) j_{k}^{p-2 r}}\right\} J_{n+\frac{1}{2}}^{\prime}\left(j_{k}\right) \\
= & \left\{(2 n-2 p-3) \sum_{r=0}^{p / 2}(-1)^{r}\left(\begin{array}{c}
p+1-r \\
r
\end{array}\right) \frac{\Gamma\left(n-r+\frac{1}{2}\right) 2^{p+1-2 r}}{\Gamma\left(n-p+r-\frac{1}{2}\right) j_{k}^{p+2-2 r}}\right. \\
& \left.-\sum_{r=1}^{\frac{p}{2}+1}(-1)^{r-1}\left(\begin{array}{c}
p+1-r \\
r
\end{array}\right) \frac{\Gamma\left(n-r+\frac{3}{2}\right) 2^{p+2-2 r}}{\Gamma\left(n-p+r-\frac{1}{2}\right) j_{k}^{p+2-2 r}}\right\} J_{n+\frac{1}{2}}^{\prime}\left(j_{k}\right) \\
= & \left\{\sum_{r=1}^{p / 2}(-1)^{r}\left(\begin{array}{c}
p+1-r \\
r
\end{array}\right) \frac{\Gamma\left(n-r+\frac{1}{2}\right) 2^{p+2-2 r}}{\Gamma\left(n-p+r-\frac{1}{2}\right) j_{k}^{p+2-2 r}} \frac{1}{(p-2 r+2)}\right. \\
& \times\left[\frac{1}{2}(2 n-2 p-3)(p-2 r+2)+r\left(n-r+\frac{1}{2}\right)\right] \\
& +\frac{\left.(2 n-2 p-3) \Gamma\left(n+\frac{1}{2}\right) 2^{p+1}-(-1)^{\frac{p}{2}}\right\} J_{n+\frac{1}{2}}^{\prime}\left(j_{k}\right) .}{\Gamma\left(n-p-\frac{1}{2}\right) j_{k}^{p+2}}
\end{aligned}
$$

Since

$$
\begin{aligned}
(n-p-3 / 2)(p-2 r+2)+r(n-r+1 / 2) & =(n-p+r-3 / 2)(p-r+2), \\
\frac{(p-r+2)}{(p-2 r+2)}\left(\begin{array}{c}
p+1-r \\
r
\end{array}\right) & =\left(\begin{array}{c}
p+2-r \\
r
\end{array}\right)
\end{aligned}
$$

and

$$
\frac{\left(n-p+r-\frac{3}{2}\right)}{\Gamma\left(n-p+r-\frac{1}{2}\right)}=\frac{1}{\Gamma\left(n-p+r-\frac{3}{2}\right)}
$$

we have

$$
\begin{aligned}
J_{n-p-\frac{5}{2}}\left(j_{k}\right)= & \left\{\sum_{r=1}^{p / 2}(-1)^{r}\left(\begin{array}{c}
p+2-r \\
r
\end{array}\right) \frac{\Gamma\left(n-r+\frac{1}{2}\right) 2^{p+2-2 r}}{\Gamma\left(n-p+r-\frac{3}{2}\right) j_{k}^{p+2-2 r}}\right. \\
& \left.+\frac{\Gamma\left(n+\frac{1}{2}\right) 2^{p+2}}{\Gamma\left(n-p-\frac{3}{2}\right) j_{k}^{p+2}}+(-1)^{\frac{p+2}{2}}\right\} J_{n+\frac{1}{2}}^{\prime}\left(j_{k}\right) \\
= & \left\{\sum_{r=0}^{(p+2) / 2}(-1)^{r}\left(\begin{array}{c}
p+2-r \\
r
\end{array}\right) \frac{\Gamma\left(n-r+\frac{1}{2}\right) 2^{p+2-2 r}}{\Gamma\left(n-p+r-\frac{3}{2}\right) j_{k}^{p+2-2 r}}\right\} J_{n+\frac{1}{2}}^{\prime}\left(j_{k}\right) .
\end{aligned}
$$

Thus, (4) is true for $p+2$. For $p+3$ we use (7), taking $\alpha=n-p+\frac{5}{2}$, and the remainder of the proof is similar.

To establish (3), we need another property of spherical Bessel functions. 
Lemma 2. Let $n$ be an integer. For all nonnegative integers $p$, we have

(8)

$$
J_{n+p+\frac{3}{2}}\left(j_{k}\right)=\left\{\sum_{r=0}^{[p / 2]}(-1)^{r+1}\left(\begin{array}{c}
p-r \\
r
\end{array}\right) \frac{\Gamma\left(n+p-r+\frac{3}{2}\right) 2^{p-2 r}}{\Gamma\left(n+r+\frac{3}{2}\right) j_{k}^{p-2 r}}\right\} J_{n+\frac{1}{2}}^{\prime}\left(j_{k}\right) .
$$

Proof. The proof is similar to that of Lemma 1 except for the next few changes. For $p=0$, we use the formula

$$
z J_{\alpha}{ }^{\prime}(z)-\alpha J_{\alpha}(z)=-z J_{\alpha+1}(z)
$$

with $\alpha=n+1 / 2$. For $p=1$, we use (7) with $\alpha=n+3 / 2$. For $p+2, p+3$, we use (7) respectively with $\alpha=n+p+\frac{5}{2}, n+p+\frac{7}{2}$.

\section{Proofs of the THEOREMS}

Proof of Theorem 1. Using Lemma 1 with $p=2 n$, we obtain

$$
J_{-\left(n+\frac{1}{2}\right)}\left(j_{k}\right)=\left\{\sum_{r=0}^{n}(-1)^{r}\left(\begin{array}{c}
2 n-r \\
r
\end{array}\right) \frac{\Gamma\left(n-r+\frac{1}{2}\right) 2^{2 n-2 r}}{\Gamma\left(-n+r+\frac{1}{2}\right) j_{k}^{2 n-2 r}}\right\} J_{n+\frac{1}{2}}^{\prime}\left(j_{k}\right) .
$$

But

$$
\Gamma\left(m+\frac{1}{2}\right)=\frac{\sqrt{\pi}(2 m) !}{2^{2 m} m !} \text { for } m=0,1,2, \ldots
$$

and

so that

$$
\Gamma\left(-m+\frac{1}{2}\right)=\frac{\sqrt{\pi}(-1)^{m} 2^{2 m} m !}{(2 m) !} \text { for } m=0,1,2, \ldots
$$

$$
\left(\begin{array}{c}
2 n-r \\
r
\end{array}\right) \frac{\Gamma\left(n-r+\frac{1}{2}\right) 2^{2 n-2 r}}{\Gamma\left(-n+r+\frac{1}{2}\right)}=(-1)^{n+r} \frac{(2 n-r) !(2 n-2 r) !}{r !\left[2^{n-r}(n-r) !\right]^{2}} .
$$

An application of the formula $[3, \S 3.12]$

$$
J_{\alpha}^{\prime}(z) J_{-\alpha}(z)-J_{\alpha}(z) J_{-\alpha}^{\prime}(z)=\frac{2 \sin (\alpha \pi)}{\pi z}
$$

gives

$$
J_{-\left(n+\frac{1}{2}\right)}\left(j_{k}\right)=\frac{2(-1)^{n}}{\pi j_{k} J_{n+\frac{1}{2}}^{\prime}\left(j_{k}\right)} .
$$

Hence, in view of (10) and (12), we obtain

$$
\left(J_{n+\frac{1}{2}}^{\prime}\left(j_{k}\right)\right)^{2}=\left(\frac{\pi}{2} \sum_{r=0}^{n} \frac{(2 n-r) !(2 n-2 r) !}{r !\left[2^{n-r}(n-r) !\right]^{2}} j_{k}^{-2 n+2 r+1}\right)^{-1}=j_{k}^{2 n-1} \lambda^{2}\left(j_{k}\right) .
$$

It remains to study the sign of $J_{n+\frac{1}{2}}^{\prime}\left(j_{k}\right)$. We have (see $[3, \S 15.22]$ )

$$
0<j_{k}<j_{k}(n+3 / 2)<j_{k+1} \quad \text { for } k=1,2, \ldots .
$$

Hence, the interval $\left(j_{k}, j_{k+1}\right)$ contains only one zero of $J_{n+\frac{3}{2}}(z)$ for $k=1,2, \ldots$, which implies

$$
\operatorname{sgn}\left(J_{n+\frac{3}{2}}\left(j_{k}\right)\right)=-\operatorname{sgn}\left(J_{n+\frac{3}{2}}\left(j_{k+1}\right)\right) \quad \text { for } k=1,2, \ldots .
$$


By (9) we have

$$
J_{n+\frac{1}{2}}^{\prime}\left(j_{k}\right)=-J_{n+\frac{3}{2}}\left(j_{k}\right) \text { for } k=1,2, \ldots,
$$

and it follows from (16) that

$$
\operatorname{sgn}\left(J_{n+\frac{1}{2}}^{\prime}\left(j_{k}\right)\right)=-\operatorname{sgn}\left(J_{n+\frac{1}{2}}^{\prime}\left(j_{k+1}\right)\right) \quad \text { for } k=1,2, \ldots
$$

which implies, in view of (14), that

$$
\begin{aligned}
J_{n+\frac{1}{2}}^{\prime}\left(j_{k}\right) & =\operatorname{sgn}\left(J_{n+\frac{1}{2}}^{\prime}\left(j_{k}\right)\right) j_{k}^{n-\frac{1}{2}} \lambda\left(j_{k}\right) \\
& =(-1)^{k-1} \operatorname{sgn}\left(J_{n+\frac{1}{2}}^{\prime}\left(j_{1}\right)\right) j_{k}^{n-\frac{1}{2}} \lambda\left(j_{k}\right) \text { for } k=1,2, \ldots .
\end{aligned}
$$

So, in order to obtain (2) for $j_{k}>0$, it suffices to prove that

$$
J_{p+1 / 2}^{\prime}\left(j_{1}(p+1 / 2)\right)<0 \text { for each nonnegative integer } p .
$$

For $p=0$, we have

$$
J_{\frac{1}{2}}(x)=\sqrt{\frac{2}{\pi x}} \sin x, \quad j_{k}\left(\frac{1}{2}\right)=k \pi, \quad k=1,2, \ldots,
$$

whence

$$
J_{\frac{1}{2}}^{\prime}\left(j_{1}(1 / 2)\right)=J_{\frac{1}{2}}^{\prime}(\pi)=-\frac{\sqrt{2}}{\pi}<0 .
$$

Suppose that (18) is true for some positive integer $p$, which implies that

$$
J_{p+\frac{1}{2}}^{\prime}(x)<0 \text { for all } x \in\left(j_{1}(p+1 / 2), j_{2}(p+1 / 2)\right) ;
$$

in particular,

$J_{p+\frac{1}{2}}\left(j_{1}(p+3 / 2)\right)<0 \quad$ since, by $(15), \quad j_{1}(p+3 / 2) \in\left(j_{1}(p+1 / 2), j_{2}(p+1 / 2)\right)$.

But, using (6), we have

$$
J_{p+\frac{3}{2}}^{\prime}\left(j_{1}(p+3 / 2)\right)=J_{p+\frac{1}{2}}\left(j_{1}(p+3 / 2)\right)<0,
$$

so that (18) holds for $p+1$ and consequently for all $p \geq 0$.

For $j_{k}<0$, we assume first that in the definition of $z^{\alpha}, \arg (z)$ has its principal value, and we suppose, as in $[3,3.62]$, that $\arg (-z)=\pi+\arg (z)$. Then we have

$$
\begin{aligned}
J_{n+\frac{1}{2}}^{\prime}\left(j_{k}\right) & =J_{n+\frac{1}{2}}^{\prime}\left(-j_{-k}\right) \\
& =-e^{\left(n+\frac{1}{2}\right) \pi i} J_{n+\frac{1}{2}}^{\prime}\left(j_{-k}\right) \\
& =e^{\left(n-\frac{1}{2}\right) \pi i}(-1)^{k}\left(j_{-k}\right)^{n-\frac{1}{2}} \lambda\left(j_{-k}\right) \\
& =(-1)^{k}\left(-j_{-k}\right)^{n-\frac{1}{2}} \lambda\left(-j_{k}\right) \\
& =(-1)^{k} j_{k}^{n-\frac{1}{2}} \lambda\left(j_{k}\right),
\end{aligned}
$$

since $\lambda\left(-j_{k}\right)=\lambda\left(j_{k}\right)$ and $J_{\alpha}(-z)=e^{\alpha \pi i} J_{\alpha}(z)$.

Proof of Theorem 2. Several details of the proof are similar to that of Theorem 1, and we omit them.

We replace $p$ by $-2 n-2$ in Lemma 2 to obtain

$$
\left(J_{n+\frac{1}{2}}^{\prime}\left(j_{k}\right)\right)^{2}=\left(\frac{\pi}{2} \sum_{r=0}^{-n-1} \frac{(-2 n-r-2) !(-2 n-2 r-2) !}{r !\left[2^{-n-r-1}(-n-r-1) !\right]^{2}} j_{k}^{2 n+2 r+3}\right)^{-1} .
$$


We have $[3, \S 15.22]$

$$
0<l_{k}<l_{k}(n-1 / 2)<l_{k+1} \quad \text { for } k=1,2, \ldots,
$$

which by virtue of (5) implies (17), where $j_{k}$ is replaced by $l_{k}$. So we have, by (19),

$$
J_{n+\frac{1}{2}}^{\prime}\left(l_{k}\right)=(-1)^{k-1} \operatorname{sgn}\left(J_{n+\frac{1}{2}}^{\prime}\left(l_{1}\right)\right) l_{k}^{-n-\frac{3}{2}} \mu\left(l_{k}\right) \text { for } k=1,2, \ldots .
$$

Thus, to establish (3) for $l_{k}>0$, we have to show that

$$
(-1)^{p+1} J_{p+\frac{1}{2}}^{\prime}\left(j_{1}(p+1 / 2)\right)<0 \text { for each negative integer } p .
$$

For $p=-1$, we have

$$
J_{-\frac{1}{2}}(x)=\sqrt{\frac{2}{\pi x}} \cos x, \quad l_{k}(-1 / 2)=(2 k-1) \pi / 2, \quad k=1,2, \ldots,
$$

whence

$$
J_{-\frac{1}{2}}^{\prime}\left(l_{1}(-1 / 2)\right)=J_{-\frac{1}{2}}^{\prime}(\pi / 2)=-\frac{2}{\pi}<0 .
$$

Assume that (21) is true for some negative integer $p$, which implies by (20) that

$$
(-1)^{p+1} J_{p+\frac{1}{2}}\left(l_{1}\left(p-\frac{1}{2}\right)\right)<0,
$$

and using (9), we obtain

$$
(-1)^{p} J_{p-\frac{1}{2}}^{\prime}\left(l_{1}(p-1 / 2)\right)=(-1)^{p+1} J_{p+\frac{1}{2}}\left(l_{1}(p-1 / 2)\right)<0 .
$$

Therefore, (21) holds for $p-1$ and consequently for all $p \leq-1$.

For $l_{k}<0$, we have

$$
\begin{aligned}
J_{n+\frac{1}{2}}^{\prime}\left(l_{k}\right) & =e^{\left(n-\frac{1}{2}\right) \pi i}(-1)^{n+k+1}\left(l_{-k}\right)^{-n-\frac{3}{2}} \mu\left(l_{-k}\right) \\
& =(-1)^{n+k}\left(-l_{-k}\right)^{-n-\frac{3}{2}} \mu\left(-l_{k}\right) \\
& =(-1)^{n+k} l_{k}^{-n-\frac{3}{2}} \mu\left(l_{k}\right) . \quad \square
\end{aligned}
$$

\section{An EXPlicit QUADRATURE FORMUla}

We are now ready to deduce the following result from Theorems B and 1.

Theorem 3. Let $n$ be a nonnegative integer. For all functions $f$ of exponential type $2 \tau$ such that

$$
x^{2 n} f(x) \in L^{1}(\mathbb{R}),
$$

we have

$$
\begin{aligned}
\int_{-\infty}^{\infty} x^{2 n} f(x) d x & \\
= & \frac{\pi}{\tau^{2 n+1}} \sum_{\substack{k=-\infty \\
k \neq 0}}^{\infty}\left(\sum_{r=0}^{n} \frac{(2 n-r) !(2 n-2 r) !}{r !\left[2^{n-r}(n-r) !\right]^{2}} j_{k}^{2 r}\right) f\left(\frac{j_{k}}{\tau}\right) \\
& +\frac{\pi}{\tau^{2 n+1}}(2 n+1)\left(\frac{(2 n) !}{2^{n} n !}\right)^{2} f(0) .
\end{aligned}
$$


Proof. Without loss of generality we may assume that $f(z)$ is even. Let

$$
g(x):=\frac{1}{x^{2}}\left[f(x)-\left(2^{n+\frac{1}{2}} \Gamma\left(n+\frac{3}{2}\right) \frac{J_{n+\frac{1}{2}}(\tau x)}{(\tau x)^{n+\frac{1}{2}}}\right)^{2} f(0)\right] .
$$

Since $f(z)$ and $J_{n+\frac{1}{2}}(z) / z^{n+\frac{1}{2}}$ are even, their derivatives vanish at zero. Besides, we have $\lim _{z \rightarrow 0} J_{\alpha}(z) / z^{\alpha}=1 /\left(2^{\alpha} \Gamma(\alpha+1)\right)$. Thus $\lim _{z \rightarrow 0} g(z)$ exists, and consequently $g(z)$ is entire. According to the hypothesis and to the formula [3, p. 405], we have

$$
\int_{-\infty}^{\infty} \frac{J_{n+\frac{1}{2}}^{2}(x)}{x} d x=\frac{2}{2 n+1}
$$

and $g(x)$ satisfies the conditions of Theorem B with $\alpha=n+\frac{1}{2}$. Therefore, we have

$$
\int_{-\infty}^{\infty} x^{2 n+2} g(x) d x=\frac{\pi}{\tau^{2 n+3}} \sum_{\substack{k=-\infty \\ k \neq 0}}^{\infty}\left(\sum_{r=0}^{n} \frac{(2 n-r) !(2 n-2 r) !}{r !\left[2^{n-r}(n-r) !\right]^{2}} j_{k}^{2 r+2}\right) g\left(\frac{j_{k}}{\tau}\right) .
$$

Replacing $g(x)$ by its value and using (24), we readily obtain (23).

Note that, in formula (54) of [1], which corresponds to (25) with $n=1$, there is a superfluous factor 32 . As a consequence of Theorem 3 we have the following

Corollary 1. If $n$ is a nonnegative integer, then for all functions $f$ of exponential type $\tau$ such that

$$
x^{n} f(x) \in L^{2}(\mathbb{R}),
$$

we have

$$
\begin{aligned}
\int_{-\infty}^{\infty} x^{2 n}|f(x)|^{2} d x= & \frac{\pi}{\tau^{2 n+3}} \sum_{\substack{k=-\infty \\
k \neq 0}}^{\infty}\left(\sum_{r=0}^{n} \frac{(2 n-r) !(2 n-2 r) !}{r !\left[2^{n-r}(n-r) !\right]^{2}} j_{k}^{2 r}\right)\left|f\left(\frac{j_{k}}{\tau}\right)\right|^{2} \\
& +\frac{\pi}{\tau^{2 n+1}}(2 n+1)\left(\frac{(2 n) !}{2^{n} n !}\right)^{2}|f(0)|^{2} .
\end{aligned}
$$

Proof. Write $f(x)=f_{1}(x)+i f_{2}(x)$, where $f_{1}(x)=\Re(f(x))$ and $f_{2}(x)=\Im(f(x))$ when $x \in \mathbb{R}$. The functions $f_{1}^{2}(x), f_{2}^{2}(x)$ satisfy the conditions of Theorem 3 . Hence, by (23), formula (26) holds for $f_{1}(x)$ and $f_{2}(x)$. The result follows since $|f(x)|^{2}=f_{1}^{2}(x)+f_{2}^{2}(x)$.

\section{REFERENCES}

1. C. Frappier and P. Olivier, A quadrature formula involving zeros of Bessel functions, Math. Comp. 60 (1993), 303-316. MR 93d:41025

2. G. R. Grozev and Q. I. Rahman, A quadrature formula with zeros of Bessel functions as nodes, Math. Comp. 64 (1995), 715-725. MR 95f:65054 
3. G. N. Watson, A treatise on the theory of Bessel functions, 2nd ed., Cambridge Univ. Press, Cambridge, 1952. MR 6:64a

Département de Mathématiques et de Statistique, Université de Montréal, Montréal, Québec, Canada H3C 3J7

E-mail address: benghanr@ere.umontreal.ca

Département de Mathématiques et de Génie Industruel, École Polytechnique, CP 6079, Succ. Centre Ville, Montréal, Québec, Canada H3C 3A7

E-mail address: frappier@mathappl.polymtl.ca 